\title{
Towards Formal Models of Blameworthiness
}

\author{
Meir Friedenberg \\ Department of Computer Science \\ Cornell University \\ meir@cs.cornell.edu
}

\begin{abstract}
As we move towards an era in which autonomous systems are ubiquitous, being able to reason formally about moral responsibility for outcomes will become more and more critical. My research has focused on formalizing notions of blameworthiness and responsibility. I summarize here some work by myself and others towards this end and also discuss interesting directions for future work.
\end{abstract}

\section{CCS CONCEPTS}

- Applied computing $\rightarrow$ Law; Psychology; • Computing methodologies $\rightarrow$ Causal reasoning and diagnostics; • Computing methodologies $\rightarrow$ Multi-agent systems.

\section{KEYWORDS}

blameworthiness; responsibility; AI in society

\section{ACM Reference Format:}

Meir Friedenberg. 2019. Towards Formal Models of Blameworthiness. In AAAI/ACM Conference on AI, Ethics, and Society (AIES '19), January 2728, 2019, Honolulu, HI, USA. ACM, New York, NY, USA, 2 pages. https: //doi.org/10.1145/3306618.3314321

\section{INTRODUCTION}

As we move towards an era in which autonomous systems are ubiquitous, being able to reason formally about moral responsibility for outcomes will become more and more critical. Unfortunately, though, pinning down any of the many notions relating to moral responsibility is notoriously difficult.

Halpern and Kleiman-Weiner [4] (HK from now on) developed a formalization of a notion they called blameworthiness that seems to capture one intuitive aspect of moral responsibility. But while the definition is compelling and behaves intuitively in many cases, there are certain important types of cases where the HK definition does not seem to adequately capture how we ascribe responsibility. In my research I've been working on developing conceptually similar approaches to blameworthiness and responsibility that work in more wide-ranging applicable scenarios.

In the following sections I first review the HK definition of blameworthiness, then summarize recent work with my advisor, Joe Halpern, extending it to multi-agent settings, and finally discuss some exciting work that we are starting to explore.

Permission to make digital or hard copies of part or all of this work for personal or classroom use is granted without fee provided that copies are not made or distributed for profit or commercial advantage and that copies bear this notice and the full citation on the first page. Copyrights for third-party components of this work must be honored.

For all other uses, contact the owner/author(s).

AIES '19, January 27-28, 2019, Honolulu, HI, USA

(c) 2019 Copyright held by the owner/author(s).

ACM ISBN 978-1-4503-6324-2/19/01.

https://doi.org/10.1145/3306618.3314321

\section{HK BLAMEWORTHINESS}

Roughly speaking, according to the HK definition, an agent is blameworthy for an outcome if there was some alternative course of action the agent could have taken that would have decreased the probability of the outcome occurring. But this doesn't tell the full story; after all, if someone could save a vase from breaking but only by giving up their own life, we would hardly consider that individual blameworthy for the vase breaking; the cost of saving it is too high. HK's definition of blameworthiness tries to balance this cost with the effect of choosing a different action.

Their definition is based on a causal models framework. For the sake of brevity, I include here only the most crucial components to understand the basics of their definition. We say that an agent's epistemic state consists of a set $\mathcal{K}$ of causal settings that describe ways the world might work and a distribution $\operatorname{Pr}$ over $\mathcal{K}$ that describes how likely each causal setting is to describe the world. We then write $\operatorname{Pr} \llbracket[A=a] \varphi \rrbracket_{\mathcal{K}}$ for the probability, given that distribution over causal settings, that outcome $\varphi$ will occur if the agent takes action $a$. We also define a function $c$ such that $c(a)$ is the agent's expected cost of taking action $a$. Given an action $a$ and an alternative action $a^{\prime}$, $\delta_{a, a^{\prime}, \varphi}^{(\operatorname{Pr}, \mathcal{K})}=\max \left(0, \operatorname{Pr} \llbracket[A=a] \varphi \rrbracket_{\mathcal{K}}-\operatorname{Pr} \llbracket\left[A=a^{\prime}\right] \varphi \rrbracket_{\mathcal{K}}\right)$ captures the extent to which alternative action $a^{\prime}$ could have prevented outcome $\varphi$. HK then define the blameworthiness of an agent who takes action $a$ for outcome $\varphi$ as $\max _{a^{\prime}} \delta_{a, a^{\prime}, \varphi}^{(P r, \mathcal{K})} \frac{N-\max \left(c\left(a^{\prime}\right)-c(a), 0\right)}{N}$, where $N$ is a context-dependent parameter used to weight the cost term.

\section{BLAMEWORTHINESS IN MULTI-AGENT SETTINGS}

As HK already note, this definition of blameworthiness seems to provide unsatisfactory results in settings where multiple agents are involved. In particular, if each agent believes that the other agents will bring about $\varphi$ independent of his or her action, then the HK framework will ascribe very little blameworthiness to each agent. In settings where the agents as a whole could have prevented outcome $\varphi$, though, this doesn't seem to be what we would want.

In work presented at AAAI this year, Joe Halpern and I proposed an approach to attributing blameworthiness to agents in multiagent settings [2]. We did this in two steps: first we showed how to ascribe blameworthiness to groups, then we showed how to divide group blameworthiness among constituent members.

For the first part, to ascribe blameworthiness to groups, we argue that we need to consider possible ways the group could have coordinated. If the group could have easily coordinate to avoid outcome $\varphi$ then we should consider them blameworthy for it occurring, but if it would have been essentially impossible for them to coordinate (e.g the group members have no means to communicate with each other) then they should have little blameworthiness. To model this, we associate with each possible way that the group can coordinate 
a distribution over how the agents will act, which in turn can be associated with a distribution over causal settings. Then by changing the cost function to give the expected cost of a group coordination and changing the $\delta$ term to be $\max \left(0, \operatorname{Pr}_{1} \llbracket \varphi \rrbracket_{\mathcal{K}}-\operatorname{Pr}_{2} \llbracket \varphi \rrbracket_{\mathcal{K}}\right)$ (considering two different possible distributions that could be induced by group coordination), we can update the definition above to capture the blameworthiness of groups.

Given a notion of group blameworthiness, we can address the next question: how can the blameworthiness of a group be apportioned among the individual agents in the group. To do this, we suggest three axioms that we believe apportionment of blame should satisfy:

- (Efficiency) The sum of the blameworthiness ascribed to the individual agents must exactly equal the blameworthiness of the entire group.

- (Symmetry) The names of agents should not affect their blameworthiness, so if we simply rename them then the blameworthiness ascribed to them should remain the same.

- (Strong Monotonicity) If an agent contributes more to the group blameworthiness of all groups in one scenario than another, then that agent also ought to have a greater degree of (personal) blameworthiness in the first scenario.

Young [5] showed that in fact the only distribution procedure that satisfies Efficiency, Symmetry, and Strong Monotonicity is the Shapley value from cooperative game theory. We thus have a technique for assigning a degree of blameworthiness for an outcome to individuals in group settings.

It's also worth noting that these two results are essentially separable: if an alternative definition of group blameworthiness is used, the Shapley value could be used to ascribe blameworthiness to individual agents as it would still be the unique procedure satisfying the desired axioms.

Finally, in settings where there is only one agent, if we assume (as HK implicitly did) that an agent can completely decide his or her own actions without the decision process itself incurring costs beyond the costs of the action, then the definition we provided in fact agrees with the HK definition.

\section{FUTURE DIRECTIONS}

There are many important directions to take this work on responsibility and blameworthiness. Here I summarize two that I'm excited to start exploring.

\subsection{Norms \& Responsibility}

Consider a scenario where a police officer and a civilian are standing by passively while a crime is committed; it seems to us that people ascribe more blameworthiness to the police officer because there is an expectation that a police officer will act to prevent a crime. Similarly, consider a case where two individuals get into a car accident because driver $A$ turned around to check on a child in the back seat and driver $B$ decided to rush through the intersection to try to beat driver $A$. American tort law considers driver $B$ to be more blameworthy due to a greater deviation from the legal norm (among other reasons in this scenario) [1]. The original HK definition and our extension to multi-agent settings do not take into account these norms and the extent to which an agent deviated from a norm. The examples above suggest that it will be important to understand the relation between norms and responsibility. To reason about responsibility in real-world scenarios it will be important to develop formal models for reasoning about blameworthiness when norms play a role. Normality has been added to the standard definition of causality in prior work [3], but combining it with blameworthiness seems subtle.

\subsection{Behavioral Studies}

A second line of work that I'm beginning to explore is trying to understand how humans really ascribe blameworthiness. How do costs of alternative actions actually affect how people ascribe blameworthiness? Are certain types of costs never considered? How does type vs. magnitude of cost play a role? To what extend does our formalization of Multi-Agent blameworthiness accord with human ascriptions? Do our axioms get obeyed? And how might autonomous systems be treated differently with regard to ascriptions of blameworthiness? I am beginning to design human behavioral experiments to explore these and other related questions.

\section{CONCLUSION}

HK and our AAAI-19 paper, combined with our work in progress on both norms and human studies, lays an important foundation on which to understand and model blameworthiness and responsibility. At the same time, there are still many questions beyond these that I think are important to explore in this domain. For example, how should we ascribe blameworthiness in settings where agents have plans and perform multiple actions in sequence rather than just taking a single action? How might the ways we ascribe responsibility affect and incentivize patterns of behavior?

I believe that being able to both prescriptively and descriptively understand ascription of responsibility and blameworthiness will be important in the coming years and that building models through which to reason about these issues will help give new insight into the beneficial adoption of autonomous systems in society.

\section{ACKNOWLEDGMENTS}

This work has been supported in part by NSF grants IIS-1703846 and IIS-1718108, ARO grant W911NF-17-1-0592, and a grant from the Open Philanthropy project.

\section{REFERENCES}

[1] American Law Institute. 2000. Restatement of the Law Third, Torts, Apportionment of Liability. American Law Institute Publishers.

[2] M. Friedenberg and J. Y. Halpern. 2019. Blameworthiness in Multi-Agent Settings. In Proceedings of the Thirty-Third AAAI Conference on Artificial Intelligence (AAAI19).

[3] J. Y. Halpern and C. Hitchcock. 2015. Graded causation and defaults. British fournal for the Philosophy of Science 66, 2 (2015), 413-457.

[4] J. Y. Halpern and M. Kleiman-Weiner. 2018. Towards Formal Definitions of Blameworthiness, Intention, and Moral Responsibility. In Proceedings of the Thirty-Second AAAI Conference on Artificial Intelligence (AAAI-18). 1853-1860.

[5] H. P. Young. 1985. Monotonic solutions of cooperative games. International fournal of Game Theory 14, 2 (1985), 65-72. 